\title{
Efeito da idade sobre a fertilidade e perdas embrionárias de éguas inseminadas com sêmen asinino diluído e resfriado a $5^{\circ} \mathrm{C}$ por 12 horas de armazenamento
}

\author{
[Effect of age on fertility and embryonic loss of mares inseminated with jackass semen \\ diluted and cooled at $5^{\circ} \mathrm{C}$ for 12 hours] \\ R. Rossi ${ }^{1}$, J.M. Silva Filho ${ }^{2}$, M.S. Palhares ${ }^{2}$, R.A. Martins ${ }^{3}$, F.R. Anjos ${ }^{4}$, M.M. Silva ${ }^{4}$ \\ ${ }^{1}$ Aluna de pós-graduação - Escola de Veterinária - EV-UFMG - Belo Horizonte, MG \\ ${ }^{2}$ Escola de Veterinária - EV-UFMG - Belo Horizonte, MG \\ ${ }^{3}$ Médico veterinário residente - EV-UFMG - Belo Horizonte, MG \\ ${ }^{4}$ Médica veterinária autônoma \\ RESUMO
}

\begin{abstract}
Estudou-se o efeito da idade sobre a fertilidade de éguas inseminadas com sêmen asinino diluído, resfriado e armazenado. Os ciclos foram acompanhados por palpação transretal e rufiação, sendo as inseminações realizadas às terças, quintas e sábados, a partir da detecção de um folículo de 3,0 a 3,5cm de diâmetro, em um dos ovários, até a ovulação. O sêmen de cinco jumentos da raça Pêga foi diluído nos diluidores de leite em pó desnatado-glicose ou glicina-gema de ovo, resfriado a $5^{\circ} \mathrm{C}$ e armazenado por 12 horas, sendo a dose inseminante de 400 × $10^{6}$ espermatozoides móveis (no momento da diluição final, pré-resfriamento). Os resultados de 195 ciclos estrais, referentes a 141 éguas, foram agrupados em classes, de acordo com a idade das éguas: 2,5 a 6 anos, 6,5 a 10 anos, 10,5 a 14 anos e 14,5 a 19 anos. As taxas de concepção, ao primeiro ciclo, foram de $68,42 \%, 50,75 \%, 46,88 \%$ e $52,17 \%$ e, após quatro ciclos, de $69,57 \%, 47,92 \%, 46,34 \%$ e $45,71 \%$ para as faixas etárias de 2,5 a 6, 6,5 a 10,10,5 a 14 e 14,5 a 19 anos, respectivamente (P>0,05). A idade não teve efeito sobre a fertilidade das éguas inseminadas com sêmen asinino diluído e resfriado.
\end{abstract}

Palavras-chave: jumento, idade, sêmen resfriado, fertilidade, perda embrionária

\begin{abstract}
The effect of the mare age on fertility of mares inseminated with diluted, cooled and stored jackass semen was studied. The females were controlled by transrectal palpation and teasing, and inseminated every Tuesday, Thursday and Saturday, since the detection of a 3.0 to $3.5 \mathrm{~cm}$ follicle diameter, in one of the ovaries, until ovulation. The semen of five Pêga jackasses was diluted in skim milk-glucose or in egg yolk-glicine extender and cooled at $5^{\circ} \mathrm{C}$ for 12 hours, with the inseminate dose of $400 \times 10^{6}$ motile spermatozoa (at the moment of the final dilution, before cooling). The results of 195 cycles of 141 mares were grouped, in accordance with the age: 2.5 to 6 years, 6.5 to 10 years, 10.5 to 14 years and 14.5 to 19 years. The pregnancy rates for the first cycle were $68.42 \%, 50.75 \%, 46.88 \%$ and $52.17 \%$, and after four cycles, the pregnancy rates/cycle were $69.57 \%, 47.92 \%, 46.34 \%$ and $45.71 \%$, respectively for 2.5 to $6,6.5$ to $10,10.5$ to 14 and 14.5 to 19 years $(P>0,05)$. The mare age had no influence on fertility, using diluted and cooled jackass semen.
\end{abstract}

Keywords: jackass, age, cooled semen, fertility, embryonic loss

\section{INTRODUÇÃO}

Diferentemente do que acontece com outras espécies criadas para o consumo humano, em que as fêmeas são abatidas ainda jovens, na espécie equina ocorre seleção genética pelo desempenho atlético nas pistas, mantendo as éguas por período prolongado em atividades esportivas sendo, assim, afastadas temporariamente da reprodução (Pycock, 2006). Em virtude do ingresso tardio na reprodução, éguas de idade avançada fazem parte de diversos rebanhos e constituem importante fonte de variação nos índices de fertilidade obtidos. O valor afetivo e financeiro do animal influencia no

Recebido em 23 de setembro de 2013

Aceito em 21 de agosto de 2014

E-mail: raissarossi@hotmail.com 
tempo de permanência no rebanho, sendo que o apego e a preferência individual por certos animais, além do valor genético continuam sendo fatores importantes na escolha de matrizes que serão utilizadas na reprodução, mesmo após os 20 anos de idade. Esta tendência é uma realidade em muitos haras brasileiros, aumentando o desafio dos veterinários em utilizar éguas cada vez mais velhas na reprodução. Por outro lado, tem sido proposto que fêmeas de menor valor, como as utilizadas na produção de muares, são mantidas no rebanho, principalmente, enquanto produzirem boas crias, independentemente da sua idade.

O efeito da idade da égua sobre a fertilidade tem sido demonstrado na literatura, sendo os piores resultados atribuídos aos animais mais velhos (Jordão e Gouveia, 1950; Woods et al., 1987; Chevalier-Clément, 1989; Fleury et al., 1989; Mattos et al., 1991; McDowell et al.,1992; Camillo et al., 1997; Valle et al., 1999; Morris e Allen, 2002; Buiten et al., 2003; Allen et al., 2006). No entanto, éguas muito jovens também podem contribuir negativamente para a fertilidade do rebanho, uma vez que a imaturidade uterina dessas está relacionada a altas taxas de perdas gestacionais e a baixas taxas de nascimento (Mitchell e Allen, 1975; Lucas et al., 1991).

Diversos fatores tem sido associados à síndrome do envelhecimento, sendo importante ressaltar o desequilíbrio endócrino com alteração da função hipotalâmica-hipofisária-gonadal (Carnevale et al., 1993, 1994), o desarranjo das estruturas do oócito (Hunter, 1990; Rambags et al., 2006), as alterações dos ambientes tubárico (Brinsko et al., 1994; Brinsko et al., 1996) e uterino (Carnevale e Ginther, 1992; LeBlanc, 2003) e as alterações de conformação vulvar (Greenhoff e Kenney, 1975; Pascoe, 1979). Estes fatores estão interligados e podem ocorrer de forma associada, em um mesmo animal, com consequente redução da eficiência reprodutiva.

Nesse sentido, foi objetivo do estudo avaliar o efeito da idade sobre a fertilidade de éguas inseminadas com sêmen asinino diluído e resfriado, em fazenda destinada à produção comercial de muares.

\section{MATERIAL E MÉTODOS}

O presente estudo foi conduzido na Fazendo do Váu, no Município de Lagoa Dourada, Minas Gerais, região sudeste do Brasil, no período de outubro de 2007 a janeiro de 2008. A unidade experimental localiza-se a 1124 metros de altitude e $20^{\circ} 54^{\prime} 50^{\prime}$ ' de latitude $\mathrm{S}$ e $44^{\circ} 4^{\prime} 40^{\prime}$ ' de longitude $\mathrm{W}$.

Foram utilizadas 141 fêmeas equinas mestiças, de 2,5 a 19 anos de idade, que resultaram em 195 ciclos estrais inseminados. As fêmeas equinas foram agrupadas em classes por idade, de acordo com a faixa etária a que pertenciam e analisadas quanto à fertilidade sendo: 2,5 a 6 anos $(\mathrm{n}=23)$, 6,5 a 10 anos $(n=96), 10,5$ a 14 anos $(n=41)$ e 14,5 a 19 anos $(\mathrm{n}=35)$.

A partir da detecção de um folículo de 2,0 a $2,5 \mathrm{~cm}$ de diâmetro, em um dos ovários, as fêmeas equinas foram examinadas diariamente por meio de palpação transretal e rufiação. As inseminações foram iniciadas quando se detectou folículo com 3,0 a $3,5 \mathrm{~cm}$ de diâmetro, em um dos ovários, sendo realizadas em dias fixos às terças, quintas e sábados (três vezes/semana), até a ovulação, sendo o sêmen depositado no corpo do útero.

Para o diagnóstico de gestação, palpações transretais e rufiações de retorno foram realizadas, a partir do $15^{\circ}$ dia pós-ovulação, sendo complementadas pelo uso de ultrassonografia, realizada a cada 14 dias (SSD500, Aloka, probe de 7,5 MHz). O diagnóstico por imagem iniciava-se num período mínimo de 11 dias pós-ovulação, sendo que cada égua positiva era submetida à, pelo menos, dois diagnósticos subsequentes, até o final da estação de monta. Das éguas que reabsorveram, apenas aquelas com período gestacional inferior aos 35 dias retornaram para o programa reprodutivo, de acordo com Penzhorn et al. (1986).

Foram utilizados cinco jumentos da raça Pêga, como doadores de sêmen, avaliados em um período anterior ao experimento para comprovação da qualidade do sêmen fresco e pós- resfriamento. O sêmen foi coletado por meio de vagina artificial modelo Hannover, as terças, quintas e sábados (três vezes/semana). Após a avaliação da motilidade e vigor espermáticos, procedeu-se a contagem das 
células espermáticas em câmara de Neubauer, para cálculo da concentração, com posterior diluição do sêmen em diluidor à base de leite em pó desnatado-glicose (Kenney et al., 1983) ou de glicina-gema de ovo (Foote, 2002). As doses inseminantes, de $20 \mathrm{~mL}$ e 400 x $10^{6}$ espermatozoides móveis, foram resfriadas a $5^{\circ} \mathrm{C}$ por 12 horas de armazenamento, em contêiner especial (Palhares, 1997).

A fertilidade das éguas não foi influenciada $(\mathrm{P}>0,05)$ pelos diluidores de sêmen utilizados, pelo número de inseminações realizadas por ciclo, pelo intervalo IA-ovulação ou pela categoria reprodutiva das éguas (Rossi, 2008). Assim, com esses fatores balanceados, foi possível analisar o efeito da idade sobre a fertilidade das fêmeas equinas, agrupando-se os dados em classes por idade, segundo a faixa etária à qual pertenciam: 2,5 a 6, 6,5 a 10, 10,5 a 14 e 14,5 a 19 anos.
A eficiência de prenhez foi calculada pela conversão de dados qualitativos em quantitativos, segundo proposta de Voss et al. (1975). Os dados foram submetidos à análise de variância utilizando-se para a comparação das médias o teste de Student-Newman-Keuls (SNK). Os demais dados quantitativos foram também submetidos à análise de variância e teste SNK. Os dados proporcionais (número de ciclos/concepção, taxa de concepção/ciclo, taxa de concepção total e ciclos/égua gestante) foram analisados por dispersão de frequência e comparados pelo teste de Qui-Quadrado para detecção de diferenças entre os grupos.

\section{RESULTADOS E DISCUSSÃO}

Observa-se, na Tab. 1, que as taxas de concepção, ao primeiro ciclo, e que as taxas de concepção/ciclo, após quatro ciclos, foram similares entre os grupos de diferentes faixas etárias $(\mathrm{P}>0,05)$.

Tabela 1. Efeito da idade sobre a fertilidade, por ciclo estral, de éguas inseminadas com sêmen asinino diluído e resfriado

\begin{tabular}{|c|c|c|c|c|c|c|c|c|c|c|}
\hline \multirow[b]{3}{*}{ Ciclo } & \multicolumn{10}{|c|}{ Idade das Éguas (anos) } \\
\hline & \multicolumn{2}{|c|}{$2,5-6,0$} & \multicolumn{2}{|c|}{$6,5-10$} & \multicolumn{2}{|c|}{$10,5-14,0$} & \multicolumn{2}{|c|}{$14,5-19,0$} & \multicolumn{2}{|c|}{ Total } \\
\hline & $\begin{array}{c}\text { № de } \\
\text { Ciclos }\end{array}$ & $\begin{array}{c}\text { Taxa } \\
\text { Concepção } \\
(\%)\end{array}$ & $\begin{array}{c}\text { № de } \\
\text { Ciclos }\end{array}$ & $\begin{array}{c}\text { Taxa } \\
\text { Concepção } \\
(\%)\end{array}$ & $\begin{array}{l}\text { № de } \\
\text { Ciclos }\end{array}$ & $\begin{array}{c}\text { Taxa } \\
\text { Concepção } \\
(\%)\end{array}$ & $\begin{array}{c}\text { № de } \\
\text { Ciclos }\end{array}$ & $\begin{array}{c}\text { Taxa } \\
\text { Concepção } \\
(\%)\end{array}$ & $\begin{array}{l}\text { № de } \\
\text { Ciclos }\end{array}$ & $\begin{array}{c}\text { Taxa } \\
\text { Concepção } \\
(\%)\end{array}$ \\
\hline 1 & $19(13)^{\mathrm{a}}$ & 68,42 & $67(34)$ & 50,75 & $32(15)$ & 46,88 & $23(12)$ & 52,17 & $141(74)$ & 52,48 \\
\hline 2 & 3(3) & 100,00 & 20(8) & 40,00 & $9(4)$ & 44,44 & $8(3)$ & 37,50 & $40(18)$ & 45,00 \\
\hline 3 & $1(0)$ & 0,00 & $8(3)$ & 37,50 & $\ldots$ & $\ldots$ & $3(1)$ & 33,33 & $12(4)$ & 33,33 \\
\hline 4 & $\ldots$ & $\ldots$ & $1(1)$ & 100,00 & $\ldots$ & $\ldots$ & $1(0)$ & 0,00 & $2(1)$ & 50,00 \\
\hline Total & $23(16)$ & 69,57 & $96(46)$ & 47,92 & $41(19)$ & 46,34 & $35(16)$ & 45,71 & 195(97) & 49,74 \\
\hline
\end{tabular}

Números entre parênteses indicam as fêmeas que conceberam

Na Tab. 2 estão apresentadas as variáveis de controle e de resultados, de acordo com Valle (1997), observando-se diferenças $(\mathrm{P}<0,05)$ entre os grupos, no que diz respeito apenas à faixa etária, como era de se esperar. Todas as demais variáveis reprodutivas foram semelhantes $(\mathrm{P}>0,05)$ entre os grupos. Também não houve influência da idade $(\mathrm{P}>0,05)$ sobre a eficiência de prenhez (Tab. 2) das éguas inseminadas.

Observou-se percentual total de perdas gestacionais de $8,51 \%(12 / 141)$, em todo o rebanho, com a seguinte distribuição por faixa etária: 2,5-6,0 anos (1/12 - 8,33\%); 6,5-10 anos (5/12- 41,67\%); 10,5-14,0 anos (1/12 - 8,33\%); 14,5-19,0 anos (5/12 - 41,67\%). Não foi possível comparar as perdas gestacionais estatisticamente, devido ao pequeno número de ocorrências nas diferentes faixas etárias estudadas. Além disso, os dados foram coletados até o final da estação de monta, não sendo possível observar aumento da incidência de perdas gestacionais, acompanhando o aumento da idade. 
Efeito da idade sobre a fertilidade...

Tabela 2. Variáveis de controle e de resultados de éguas inseminadas com sêmen asinino diluído e resfriado, agrupadas de acordo com a idade

\begin{tabular}{lcccc}
\hline \multirow{2}{*}{ Variável } & \multicolumn{4}{c}{ Idade das Éguas (anos) } \\
\cline { 2 - 5 } & $2,5-6,0$ & $6,5-10$ & $10,5-14$ & $14,5-19$ \\
\hline № de ciclos & 23 & 96 & 41 & 35 \\
Idade das éguas (anos) & $4,17 \pm 1,33^{\mathrm{d}}$ & $7,71 \pm 1,00^{\mathrm{c}}$ & $12,34 \pm 0,93^{\mathrm{b}}$ & $16,07 \pm 1,22^{\mathrm{a}}$ \\
№ de IA/ciclo & $1,91 \pm 1,20$ & $1,96 \pm 0,79$ & $1,95 \pm 0,95$ & $2,17 \pm 1,10$ \\
Volume de sêmen (mL) & $3,03 \pm 1,39$ & $2,84 \pm 1,74$ & $3,03 \pm 1,75$ & $2,93 \pm 1,31$ \\
Volume de diluidor (mL) & $16,97 \pm 1,39$ & $17,17 \pm 1,75$ & $16,99 \pm 1,76$ & $17,07 \pm 1,31$ \\
Tempo colheita/diluição (min) & $19,64 \pm 3,98$ & $18,84 \pm 2,97$ & $19,03 \pm 3,38$ & $18,83 \pm 2,76$ \\
Tempo colheita/resf. (min) & $24,82 \pm 3,29$ & $24,64 \pm 4,19$ & $24,39 \pm 3,41$ & $24,07 \pm 3,06$ \\
Tempo colheita/abertura (h) & $13,15 \pm 0,68$ & $13,16 \pm 0,76$ & $13,10 \pm 0,76$ & $13,25 \pm 0,90$ \\
Tempo colheita/IA (h) & $13,30 \pm 0,69$ & $13,32 \pm 0,75$ & $13,25 \pm 0,78$ & $13,41 \pm 0,94$ \\
№ de IA/ciclo positivo & $1,88 \pm 1,36$ & $1,98 \pm 0,68$ & $1,84 \pm 0,90$ & $2,19 \pm 1,05$ \\
№ de IA/ciclo negativo & $2,00 \pm 0,82$ & $1,94 \pm 0,89$ & $2,05 \pm 1,00$ & $2,16 \pm 1,17$ \\
№ de ciclos/concepção & 1,44 & 2,09 & 2,16 & 2,19 \\
Taxa de concepção/ciclo & 0,7 & 0,48 & 0,46 & 0,46 \\
№ de ciclos/égua gestante & $1,19 \pm 0,40$ & $1,37 \pm 0,71$ & $1,21 \pm 0,42$ & $1,31 \pm 0,60$ \\
Eficiência de Prenhez & $6,70 \pm 4,58$ & $4,44 \pm 4,75$ & $4,44 \pm 4,87$ & $4,29 \pm 4,81$ \\
\hline Médias segury
\end{tabular}

Médias seguidas por letras diferentes na mesma linha, diferem entre si $(\mathrm{P}<0,05)$

No presente estudo, a idade não influenciou $(\mathrm{P}>0,05)$ a fertilidade das fêmeas equinas inseminadas com sêmen asinino diluído e resfriado. Deve-se considerar que o número de ciclos para a faixa de 2,5-6,0 foi abaixo do ideal, tanto para o primeiro ciclo, quanto para o total de ciclos, e que o teste de qui-quadrado utilizado pode causar sobreposição dos intervalos de confiança. O mesmo ocorre para a faixa de 14,519,0 anos, apenas nos dados do primeiro ciclo. No entanto, a idade é um fator de extrema importância a ser considerado quando se avaliam resultados de fertilidade, notadamente no que se refere às perdas embrionárias e fetais, tanto em éguas mais velhas (Vanderwall, 2011), quanto em fêmeas equinas muito jovens (Lucas et al., 1991; Mitchell e Allen, 1975).

Resultados de diferentes estudos divergem quanto à idade em que se observa início do declínio da fertilidade, em fêmeas equinas. Jordão e Gouveia (1950) verificaram redução da fertilidade em éguas a partir dos 10 anos de idade e McDowell et al. (1992) e Barron (1995), aos 11 anos de idade. Mattos et al. (1991) verificaram ser a taxa de gestação inversamente proporcional à idade da égua, com redução pronunciada da fertilidade aos 13 anos. Silva (1991), a partir da mesma faixa etária, observou aumento de patologias do sistema genital nas fêmeas equinas. Além disso, de acordo com a autora, observou-se maior somatório de transtornos ligados ao envelhecimento, em um mesmo animal, acompanhando o aumento da idade. Carnevale e Ginther (1992), Camillo et al. (1997) e Valle et al. (1999) observaram fertilidade inferior para éguas acima de 15 anos. Já Woods et al. (1987) observou redução aparente da fertilidade a partir dos 18 anos. Em um único estudo conduzido por Brandão (2001), similarmente ao que foi observado no presente experimento, não foram observadas diferenças de fertilidade entre as diferentes idades $(\mathrm{P}>0,05)$, o que contrasta com a literatura consultada.

Carnevale et al. (1997) observaram menor atividade folicular no grupo de éguas velhas, em comparação ao de jovens, sendo que três de nove éguas, com idade acima de 20 anos, sequer apresentaram crescimento folicular acima de $20 \mathrm{~mm}$ de diâmetro. Demonstrou-se que a senescência ocorreu em éguas acima de 20 anos de idade. No presente estudo, apenas cinco éguas tinham idade superior a 16 anos, sendo que uma, duas e duas apresentavam idades de 19,18 e 17 anos, respectivamente, com média de 16,07士1,22 anos. O pequeno número de éguas mais velhas e nenhuma no período de senescência, segundo a literatura, pode ter influenciado na obtenção de taxas de concepção similares entre as faixas etárias. 
A utilização de boas práticas de manejo e higiene utilizadas, dando enfoque ao uso da IA com sêmen diluído em diluidores apresentando antibióticos em sua formulação, podem ter favorecido os resultados do presente estudo, notadamente no que se refere às éguas mais velhas e com problemas reprodutivos. Sustentação para esta proposta tem sido encontrada na literatura, como pode ser observado nos estudos realizados por Mattos et al. (1996) e Blanchard et al. (2004), nos quais foi observada superioridade da IA em relação a monta natural, no que se refere as taxas de gestação obtidas. Doig et al. (1981), também utilizando a IA, otimizaram as taxas de nascimento de potros, excetuando-se as éguas com danos severos no endométrio. $\mathrm{O}$ fracionamento do sêmen com sua diluição em diluidores que contenham antibióticos, para a produção das doses inseminantes, reduzem o número de contaminantes inoculados no útero e o desafio do ambiente uterino, principalmente em éguas susceptíveis, podendo ser as taxas de concepção superiores em até $30 \%$ com o uso da IA, em relação à monta natural (Pickett e Shiner, 1994).

Finalmente vale salientar que, em diversos estudos, a atuação eficiente do médico veterinário, aliada ao descarte dos animais de baixa fertilidade, influenciou positivamente os índices reprodutivos obtidos (Von Lepel, 1975; Morris e Allen, 2002; Blanchard et al., 2004). No presente estudo utilizou-se rebanho de éguas mestiças visando à produção comercial de muares, com baixo valor individual se comparado ao valor de éguas oriundas de raças puras, o que poderia ter facilitado descartes mais frequentes das fêmeas. Sendo assim, ficariam no rebanho as fêmeas mais férteis, independentemente da idade. Tal proposta merecerá uma avaliação crítica em outros estudos realizados em diferentes propriedades, com o mesmo sistema de criação.

\section{CONCLUSÕES}

A idade não influenciou a fertilidade das éguas inseminadas com sêmen asinino diluído e resfriado. Assim deve-se realizar, sempre que possível, seleção do rebanho com enfoque na fertilidade. Aditivamente a um melhor controle reprodutivo das éguas, deve-se optar pela utilização de boas práticas de manejo e de higiene, com ênfase na utilização da IA com sêmen diluído em diluidores adequados, com o intuito de aumentar a eficiência reprodutiva, principalmente em éguas susceptíveis e de idade avançada.

\section{AGRADECIMENTOS}

À FAPEMIG, pelo financiamento parcial deste trabalho; ao Sr. Renato Resende pela cessão dos animais e instalações da Fazenda do Vau; à Hertape Calier e à Dra. Marcela Roscoe, pelos medicamentos concedidos para o trabalho.

\section{REFERÊNCIAS}

ALLEN, W.R.; WILSHER, S.; MORRIS, L. et al. Re-establishment of oviducal patency and fertility in infertile mares. Anim. Reprod. Sci., v.94, p.242-243, 2006.

BARRON, J.K. The effect of maternal age and parity on the racing performance of thoroughbred horses. Equine Vet. J., v.27, p.73-75, 1995.

BLANCHARD, T.L.; THOMPSON, J.A.; BRINSKO, S.P. et al. Mating mares on foal heat: a five-year retrospective study. In: Annual Convention of the American Association of Equine Practitioners, 50, 2004, Denver, Colorado, (Ed.). Plublisher: American Association of Equine Practitioners, Lexington KY. Internet Publisher: International Veterinary Information Service, Ithaca NY. Disponível em: www.ivis.org. Acessado em: 17 abr. 2008.

BRANDÃO, F.Z. Efeito da concentração espermática e do intervalo entre as duas últimas inseminações sobre a fertilidade de éguas inseminadas com sêmen a fresco diluído. 2001. 94f. Dissertação (Mestrado em Medicina Veterinária) - Escola de Veterinária Universidade Federal de Minas Gerais, Belo Horizonte, MG.

BRINSKO, S.P.; BALL, B.A.; MILLER, P.G. In vitro development of day 2 embryos obtained from young, fertile mares and aged, subfertile mares. J. Reprod. Fertil., v.102, p.371-378, 1994.

BRINSKO, S.P.; IGNOTZ, G.G.; BALL, B.A. et al. Characterization of polypeptides synthesized and secreted by oviductal epithelial cell explants obtained from young, fertile and aged, subfertile mares. Amer. J. Vet. Res., v.57, p.1346-1353, 1996. 
BUITEN, A.V.; WESTERS, P.; COLENBRANDER, B. Male, female and management risk factors for non-return to service in dutch mares. Prev. Vet. Med., v.61, p.17-26, 2003.

CAMILLO, F.; MARMORINI, P.; ROMAGNOLI, S. et al. Fertility of the first post partum estrous compared with fertility at the following estrous cycles in foaling mares and with fertility in nonfoaling mares. J. Equine Vet. Sci., v.17, p.612-616, 1997.

CARNEVALE, E.M.; BERGFELT, D.R.; GINTHER, O.J. Aging effects on follicular activity and concentrations of $\mathrm{FSH}, \mathrm{LH}$, and progesterone in mares. Anim. Reprod. Sci., v.31, p.287-299, 1993.

CARNEVALE, E.M.; BERGFELT, D.R.; GINTHER, O.J. Folicular activity and concentrations of FSH and LH associated with senescence in mares. Anim. Reprod. Sci., v.35, p.231-246, 1994.

CARNEVALE, E.M.; GINTHER, O.J. Relationship of age to uterine function and reproductive efficiency in mares. Theriogenology, v.37, p.1101-1115, 1992.

CARNEVALE, E.M.; HERMENET, M.J.; GINTHER, O.J. Age and pasture effects on vernal transition in mares. Theriogenology, v.47, p.1009-1018, 1997.

CHEVALIER-CLÉMENT, F. Pregnancy loss in the mare. Anim. Reprod. Sci., v.20, p.231-244, 1989.

DOIG, P.A.; MCKNIGHT, J.D.; MILLER R.B. The use of endometrial biopsy in the infertile mare. Canadian Vet. J., v.22, p.72-76, 1981.

FLEURY, J.J.; COSTA NETO, J.B.; ALVARENGA, M.A. Results from a embryo transfer programe with Mangalarga in Brazil. Equine Vet. J., v.8 , p.73-74, 1989.

FOOTE, R.H. Whithin-Herd use of boar semen at $5^{\circ} \mathrm{C}$, with a note eletronic monitoring of oestrus. Reprod. Dom. Anim., v.37, p.61-63, 2002.

GREENHOFF, G.R.; KENNEY, R.M. Evaluation of reproductive status of nonpregnant mares. $J$. Amer. Vet. Med. Associat., v.167, p.449-58, 1975.

HUNTER, R.H.F. Gamete lifespans in the mare's genital tract. Equine Vet. J., v.22, p.378379, 1990.
JORDÃO, P.; GOUVEIA, P.F. Eficiência reprodutiva do Puro Sangue Inglês em São Paulo. Bol. da Ind. Anim., v.11, p.23-55, 1950.

KENNEY, R.M.; HURTGEN, J.P.; PIERSON, R. et al. Society for theriogenology manual for clinical fertility evaluation of the stallion. Hastings: Society for Theriogenology, 1983, $100 \mathrm{p}$.

LEBLANC, M.M. Persistent mating induced endometritis in the mare: pathogenesis, diagnosis and treatment. Publisher: International Veterinary Information Service. 2003. Disponível em: <www.ivis.org>. Acessado em: 17 abr. 2008.

LUCAS, J.; RAESIDE, I.; BETTERIDGE, K.J. Non-invasive assessment of the incidences of pregnancy and pregnancy loss in feral horses of Sable Island. J. Reprod. Fertil., v.44 , p.479-488, 1991.

MATTOS, R.C.; CAVALHEIRO, E.P.; MATTOS, R.C. et al. Monta natural e inseminação artificial com sêmen fresco diluído em éguas Árabe. Arq. Fac. Vet. UFRGS, v.24, p.57-64, 1996.

MATTOS, R.C.; ROCHA, A.; GREGORY, R.M. et al. Relação entre aspectos clínicos e taxas de gestação no cio do potro. In: CONGRESSO BRASILEIRO DE REPRODUÇÃO ANIMAL, 9, 1991, Belo Horizonte. Anais. Belo Horizonte: Colégio Brasileiro de Reprodução Animal., 1991. p.469.

MCDOWELL, K.J.; POWELL, D.G.; BAKER, C.B. Effect of book size and age of mare and stallion on foaling rates in thoroughbred horses. J. Equine Vet. Sci., v.12, p.364-367, 1992.

MITCHELL, D.; ALLEN, W.R. Observations on reproductive performance in the yearling mare. $J$. Reprod. Fertil., v.23, p.531-536, 1975.

MORRIS, L.H.A.; ALLEN, W.R. Reproductive efficiency of intensively managed thoroughbred mares in newmarket. Equine Vet. J., v.34, p.5160, 2002.

PALHARES, M.S. Influência de um novo container para o transporte do sêmen equino diluído $e$ resfriado: $I$ - Características termodinâmicas $e$ funcionais, II - Desempenho reprodutivo de éguas inseminadas. 1997.245f. Dissertação (Doutorado em Ciência Animal) - Escola de Veterinária, Universidade Federal de Minas Gerais, Belo Horizonte, MG. 
PASCOE, R.R. Observations on the length and angle of declination of the vulva and its relation to fertility in the mare. J. Reprod. Fertil., v.27, p.299-305, 1979.

PICKET, B.W.; SHINER, K.A. Recent developments in artificial insemination in horses. Livest. Production Sci., v.40, p.31-36, 1994.

PENZHORN, B.L.; BERTSCHINGER, H.J.; COUBROUGH, R.I. Reconception of mares following termination of pregnancy with prostaglandin F2 $\alpha$ before and after day 35 of pregnancy. Equine Vet. J., v.18, p.215-217, 1986.

PYCOCK, J.F. How to maximize the chances of breeding successfully from the older maiden mare. In: Annual Convention of the AAEP, 2006, San Antonio. Proceedings. San Antonio, 2006. v.52, p.245-249.

RAMBAGS, B.P.B.; VAN BOXTEL, D.C.J.; THARASANIT, T. et al. Maturation in vitro leads to mitochondrial degeneration in oocytes recovered from aged but not young mares. Anim. Rep. Sci., v.94, p.359-361, 2006.

ROSSI, R. Comparação de dois diluidores na fertilidade de éguas inseminadas com sêmen asinino a fresco ou resfriado. 2008. 209f. Dissertação (Mestrado em Medicina Veterinária) - Escola de Veterinária, Universidade Federal de Minas Gerais, Belo Horizonte, MG.

SILVA, M.I.F. Alterações morfológicas em útero, tubas e ovários da égua. 1991. 253p. Dissertação (Mestrado em Medicina Veterinária) - Escola de Veterinária, Universidade Federal de Minas Gerais, Belo Horizonte, MG.
VALLE, G.R. Efeito da rufiação e manipulação do sistema genital e transporte de sêmen, em container "celle" modificado, na avaliação da técnica de inseminação artificial de equinos. 1997. 349f. Dissertação (Mestrado em Medicina Veterinária) - Escola de Veterinária, Universidade Federal de Minas Gerais, Belo Horizonte, MG.

VALLE, G.R.; SILVA FILHO, J.M.; OLIVEIRA, H.N. et al. Efeito da idade sobre a fertilidade de éguas inseminadas com sêmen diluído, resfriado a $14^{\circ} \mathrm{C}$ e transportado. Rev. Bras. Zootec., v.28, p.1031-1036, 1999.

VANDERWALL, D.K. Early embryonic loss. In: MCKINNON, A.O.; SQUIRES, E.L.; VAALA, W.E.; VARNER, D.D. Equine Reproduction. 2.ed., Wiley-Blackwell, 2011. p.2118-2122.

VON LEPEL, J. FRHR. Maintenance of fertility in the horse including artificial insemination. Equine Vet. J., v.7, p.97-101, 1975.

VOSS, J.L.; PICKETT, B.W.; BACK, D.G. et al. Effect of rectal palpation on pregnancy rate of nonlactanting, normaly cycling mares. J. Anim. Sci., v.41, p.829-834, 1975.

WOODS, G.L.; BAKER, C.B.; BALDWIN J.L. et al. Early pregnancy loss in brood mares. $J$. Reprod. Fertil., v.35, p.455-459, 1987. 